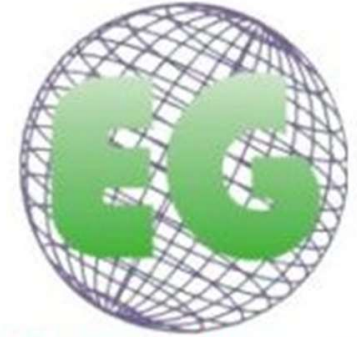

ISSN 1695-6141 $N^{\circ} 60$

\title{
Diseño y validación de la escala de Inteligencia Espiritual en la práctica sanitaria, Ica-Perú
}

Design and validation of the spiritual intelligence scale in health practice, Ica-Peru

\section{Bladimir Becerra Canales ${ }^{1}$ \\ Domizbeth Becerra Huaman²}

\begin{abstract}
1 Doctor en Salud Pública. Coordinador Regional de Gestión de la Calidad en Salud de la Dirección Regional de Salud Ica, Perú. Docente en la Universidad Autónoma de Ica, Perú. icapredica@gmail.com 2 Estudiante de Medicina Humana. Sociedad Científica de Estudiantes de Medicina de Ica (SOCEMI). Perú. Universidad Nacional San Luis Gonzaga de Ica, Perú.
\end{abstract}

\section{https://doi.org/10.6018/eglobal.417371}

Recibido: 5/03/2020

Aceptado: 6/05/2020

\section{RESUMEN:}

Introducción: La inteligencia espiritual es la única alternativa para humanizar los servicios de salud en los tiempos actuales de crisis; de ahí, que urge la necesidad de cultivarla y desarrollarla en las organizaciones de salud.

Objetivos. Diseñar y validar una escala para medir el perfil de inteligencia espiritual en la práctica sanitaria (EIEps), en una muestra de trabajadores de salud de Ica, Perú.

Materiales y métodos: Se realizó un estudio de diseño de validación de instrumentos documentales de tipo observacional y analítico, en 288 trabajadores de salud, el proceso incluye dos fases: Cualitativa (Creación del instrumento) y cuantitativa (Evaluación de sus propiedades métricas).

Resultados: El instrumento consta de 18 items, distribuidos en tres dimensiones, el análisis de fiabilidad presentó un coeficiente Alfa de Cronbach de 0,90; con valores de correlación ítem-total de 0,45 hasta 0,72 ; el análisis factorial confirmatorio mostró una estructura de tres dominios que explicaba el $54 \%$ de la varianza total. El análisis bidimensional demostró correlación significativa $(p=0,00)$ y directa, entre la Dimensión I (Vivencia espiritual en la práctica), Dimensión II (Pensamiento existencial) y la Dimensión III (Consciencia trascendental).

Conclusión: La EIEps, presentó una buena consistencia interna, con correlaciones moderadas y significativas entre sus ítems y constituye un instrumento que puede ser usado para medir el perfil de inteligencia espiritual en trabajadores de organizaciones de salud.

Palabras clave: Estudio de validación; Inteligencia Espiritual; Inteligencia; Espiritualidad; Escala.

\section{ABSTRACT:}

Introduction: Spiritual intelligence is the only alternative to humanize health services in the current time of crisis; the urgent need to cultivate and develop it in the health organizations.

Objectives: To design, and validate a scale to measure the profile of spiritual intelligence in health practice (EIEps), in a sample of healths works from Ica, Peru.

Materials and methods: A validation design study of observational and analytical documentary instruments was carried out in 288 health workers; the process includes two phases: Qualitative (creation of the instrument) and quantitative (evaluation of its metric properties). 
Results: The instrument consists of 18 items, distributed in three dimensions; the reliability analysis presented a Cronbach Alpha coefficient of 0.90 ; with item-total correlation values of 0.45 to 0.72 ; the confirmatory factor analysis showed a three-domain structure that explained $54 \%$ of the total variance. The two-dimensional demostration analysis showed significant $(p=0.00)$ and direct correlation between Dimension I (Spiritual experience in practice), Dimension II (Existential Thought) and Dimension III (Transcendental Consciousness).

Conclusion: The ElEps presented a good internal consistency, with moderate and significant correlations between its items and constitutes an instrument that can be used to measure the profile of spiritual intelligence in health organization workers.

Key words: Validation study; Spiritual Intelligence; Intelligence; Spirituality; Scale.

\section{INTRODUCCIÓN}

En la década de los 80 , apareció una teoría revolucionaria respecto a la inteligencia humana: las inteligencias múltiples de Gardner ${ }^{(1,2)}$; este científico norteamericano con un criterio multidimensional define la inteligencia como la habilidad para resolver un problema, producir un producto o realizar un servicio valorado en un contexto cultural; sostiene que no se trata de cuantificar la inteligencia, sino cómo manifestamos nuestra inteligencia, quedando desestimado el enfoque unidimensional que cuantificaba la inteligencia con un número. Entonces propone nueve inteligencias o capacidades consideradas como vías relevantes de adaptación exitosa al medio: lingüística, musical, lógico-matemática, espacial, cinestésico-corporal, intrapersonal, interpersonal, naturalista y existencial o espiritual. No obstante, es necesario señalar aquí la etimología del término: la palabra latina intelligentia proviene de intelligere, vocablo compuesto por los términos intus (entre) y legere (escoger o leer). Es decir, etimológicamente la inteligencia sería la capacidad de saber escoger la mejor alternativa, pero también la capacidad de saber leer la realidad de la manera más completa y profunda posible ${ }^{(3)}$.

Desde entonces, la novena inteligencia múltiple o Inteligencia Espiritual (IE), ha sido estudiada en los últimos años por diversos investigadores (4-10). La definición primigenia de IE, hace referencia a la capacidad de situarse uno mismo frente a facetas más extremas del cosmos, lo infinito y la capacidad de preguntarse por determinadas características existenciales de la condición humana, como el significado de la vida y de la muerte, el destino final del mundo físico, el mundo psicológico y la posibilidad de experimentar algunas emociones especiales, como un profundo amor o la contemplación artística ${ }^{(1)}$.

En un contexto más actual, King (11) define la IE como un conjunto de capacidades mentales que contribuyen a la sensibilización, la integración y adaptación de los aspectos no materiales y trascendente de la existencia del ser humano, lo que conlleva a una profunda reflexión existencial, de mejora de significado, el reconocimiento de un ser trascendente, y el dominio de los estados espirituales. En ese sentido considera que tal inteligencia capacita para el pensamiento existencial y crítico, faculta para contemplar críticamente la naturaleza de la existencia, la realidad, el universo, el espacio y el tiempo. Esta definición se condice con el enfoque de Gardner (2), quien también considera que de existir una inteligencia espiritual, la misma debiera llamarse más bien filosófica o existencial, ya que facultaría al ser humano para situarse a sí mismo con respecto al cosmos y buscar respuestas a los grandes interrogantes existenciales.

La IE, nos ayuda a encontrar el sentido de la existencia humana y el significado de la 
vida (12,13); de ahí, que la mayoría de autores, atribuye a esta inteligencia, la capacidad de buscar sentido, significado y propósito en la vida y en el trabajo, para descubrir interconexiones entre las diversas dimensiones de la existencia y el uso de este conocimiento para resolver problemas cotidianos, buscando una vida mejor (14).

Por su parte, Kwilecki (15), aplicó el modelo de Emmons (16), a un estudio de caso, confirmando empíricamente la observación de conductas ajustables a las dimensiones propuestas por este investigador, en sus hallazgos pone énfasis en la capacidad para la resolución de problemas que mostró el individuo a partir de conductas asociadas a este modelo de inteligencia espiritual.

En ese contexto y con una visión más consciencial ${ }^{(17,18)}$, la IE es definida por el autor, como la conjunción de capacidades mentales que se ocupan de cuestiones existenciales, trascendentes y/o conscienciales de la condición de ser-humano y su conexión con el universo; para desarrollar facultades que conducen a la sensibilización, humanización y autorrealización del ser humano auténticamente genuino; de ahí, que esta modalidad de inteligencia debiera llamarse también Inteligencia Consciencial (IC); porque le permite al hombre reflexionar sobre sí mismo, ver el mundo material desde otras perspectivas, facilita el autoconocimiento y permite crear consciencia. En ese orden de ideas, señala que la Inteligencia Espiritual en la Practica Sanitaria (IEPS), es la capacidad de saber escoger a la consciencia sobre el ego, como el verdadero conductor de la vida, extrayendo su sabiduría para encontrar soluciones espirituales a los problemas en el quehacer de la práctica en salud.

Desarrollar y cultivar IE O IC en las organizaciones de salud, permitiría formar servidores públicos de calidad humana con altos niveles de humanización, reflejados en sus comportamientos virtuosos (Compasión, trato digno, empatía, solidaridad, respeto, responsabilidad, honestidad, entre otros) y en consecuencia tener establecimientos sanitarios de calidad y un sistema de salud más efectivo.

En las últimas décadas se han desarrollado varios instrumentos dirigidos a medir la espiritualidad(19-21), sin embargo, la mayoría de estos instrumentos fueron desarrollados en idioma inglés y en poblaciones diferentes.

Por su parte, King y DeCicco, desarrollaron un modelo, el resultado de este estudio se tradujo en un nuevo constructo para evaluar y medir el nivel de inteligencia espiritual, el autoinforme de inteligencia espiritual Inventario-24, SISRI-24 (7).

Psicólogos, como Wolman (9) y Amram (22), argumentan que la IE promueve el desarrollo de Potencial humano, en consecuencia es necesario volverlo operativo; es decir, contextualizarlo en diversos escenarios, como es el caso del ámbito sanitario.

Considerando este planteamiento el objetivo del estudio, fue diseñar y validar una escala para medir el perfil de inteligencia espiritual en el contexto de la práctica sanitaria; en una muestra de trabajadores de salud de Ica, Perú. 


\section{MÉTODO}

\section{Diseño y población de estudio}

Reuniendo los elementos considerados hasta aquí, se planteó un estudio de tipo transversal de diseño validación de instrumentos documentales, se realizó durante los meses de septiembre a diciembre del año 2019, involucrando a trabajadores de Hospitales y Centros de Salud de la provincia de Ica. Se estimó un tamaño muestral mínimo para validar una escala de 18 ítems en 288 participantes, considerando por lo menos tener 16 unidades muestrales por ítem ${ }^{(23)}$, las mismas que fueron seleccionadas por disponibilidad de tipo no probabilístico. Se incluyeron a trabajadores de áreas administrativas y asistenciales que se encuentren en el momento de la aplicación del instrumento y que deseen participar en el estudio; se excluyeron trabajadores ausentes y que no deseaban participar en la investigación.

\section{Desarrollo y validación de la escala}

Para medir el perfil de IE en trabajadores de organizaciones de salud, se construyó una escala que Ilamamos Escala de Inteligencia Espiritual en la Practica Sanitaria (EIEps), según un modelo teórico propio, basado en las publicaciones previas ${ }^{(4-18)}$.

El procedimiento de diseño y validación se realizó en dos fases ${ }^{(24,25)}$ :

Fase cualitativa: Corresponde a la creación del instrumento (Validez de contenido), incluye: a) Revisión del concepto de la literatura: El cual consistió en la revisión del conocimiento disponible que se tiene hasta el momento respecto al concepto de la variable IE en el contexto de la práctica sanitaria, concluyendo que la misma se encontraba parcialmente definida. b) Formulación de los ítems del instrumento: Teniendo en cuenta la circunstancia que el concepto de la variable se encontraba parcialmente definida, algunos ítems de los 56 iniciales se formularon a partir de la revisión de la literatura y/o modificando reactivos de otros instrumentos. c) Evaluación del contenido por jueces: Se eligió a un grupo multidisciplinario de jueces (05 profesionales) pertenecientes a distintos campos del conocimiento a fin de evitar percepciones sesgadas y opiniones subjetivas acerca del tema en proceso de evaluación; la tarea de los jueces fue revisar los ítems ya construidos en términos de relevancia, suficiencia, pertinencia y claridad con la que están redactados, lo que permitió reducir el número de ítems del instrumento a 38 reactivos. d) Prueba piloto: El instrumento así construido fue aplicado a 288 trabajadores de salud; en esta fase se volvió a evaluar la claridad con la que están redactados los ítems, mejorando la sintaxis y semántica de alguno de ellos.

Fase cuantitativa: Corresponde a la evaluación de las propiedades métricas, incluye: a) Análisis de la consistencia: Se procedió a explorar la correlación de cada uno de los ítems respecto del total, para evaluar la idoneidad de estos ítems según lo cual se quedan o no en el instrumento. b) Análisis de la variabilidad y matriz de correlación: En esta fase de la reducción de los ítems, fueron eliminados 10 ítems más, de tal modo que nos quedamos únicamente con 28 , para tal efecto se ordenaron todos los ítems según el índice de correlación que guardan con el resultado total de mayor a menor, de tal modo que los últimos ítems (6), que tuvieron la menor magnitud de correlación con el puntaje total y aquellos que expresaron menor grado de variabilidad fueron eliminados. c) Análisis Factorial: el Análisis Factorial Exploratorio incluyó el test 
de esfericidad de Bartlett; la medida de adecuación muestral KMO; para confirmar si procede su factorización, en este procedimiento se perdieron 4 ítems más y, finalmente, el Análisis factorial Confirmatorio reveló una estructura de tres dimensiones.

\section{Análisis estadísticos}

Se describieron las características de la población objeto de estudio mediante frecuencias absolutas y relativas para las variables categóricas, así como medidas de tendencia central y dispersión para las variables numéricas.

Se realizó un análisis de la variabilidad de los ítems, considerándose como elevados los valores superiores a 0,53 ; dado que, por tratarse de una escala de tipo Likert, se acepta, al igual que este autor, un valor mínimo de 0,20 para poder afirmar que los ítems discriminan (26); así como un análisis de la matriz de correlación de los ítems e ítem total, mediante el coeficiente de correlación de Pearson (considerándose como apropiados los valores superiores a 0,50). Previo al análisis factorial, se determinó la existencia de relaciones significativas entre las variables, con el Test de Esfericidad de Bartlett y el test de Kaiser-Meyer-Olkin (KMO), tomando como un correcto ajuste de la muestra promedio y por ítem cuando los valores fueron superiores a $0,70{ }^{(27)}$.

Se incluyó un análisis de fiabilidad para escalas y la consistencia interna fue medida con el Alpha de Cronbach; garantizando que estos índices superen el mínimo recomendado por la literatura $(0,70)$ para ser considerado un instrumento confiable ${ }^{(28)}$; Se hicieron correlaciones la variable IE con sus dimensiones, considerando un intervalo de confianza al $95 \%$ y a todo valor de $p<0,05$ como estadísticamente significativo. Para el procesamiento de los datos se usó el paquete estadístico "Statistical Package for the Social Sciences" para Windows versión 24.0 en español.

\section{Aspectos éticos}

Se solicitó el permiso institucional y se indicó a los encuestados sobre el propósito de la investigación y el carácter voluntario y anónimo de su colaboración. Se destacó que la participación podía interrumpirse en el momento en que el participante lo deseara. Se obtuvo el consentimiento verbal y no se ofreció ningún tipo de contraprestación.

\section{RESULTADOS}

La mayoría de trabajadores de salud, eran de sexo femenino (57,6\%); condición laboral nombrado $(65,6 \%)$; asistenciales $(77,1 \%)$ y procedían de centros de salud $(54,2 \%)$; el promedio de edad fue de 36 años. (Tabla 1 ).

Tabla 1: Caracterización de los trabajadores de salud.

\begin{tabular}{llcc}
\hline \multicolumn{2}{c}{ Variables de caracterización } & $\mathrm{F}$ & $\%$ \\
\hline Sexo & Masculino & 122 & 42,4 \\
& Femenino & 166 & 57,6 \\
Condición laboral & Nombrado & 189 & 65,6 \\
& Contratado & 99 & 34,4 \\
Grupo & Administrativo & 66 & 22,9 \\
ocupacional & Asistencial & 222 & 77,1
\end{tabular}




\begin{tabular}{clcc} 
Centro laboral & $\begin{array}{l}\text { Hospitales } \\
\text { Centros de }\end{array}$ & 132 & 45,8 \\
& Salud & 156 & 54,2 \\
Edad en años & Media \pm DE & $35,66 \pm 10,75$ \\
\hline F= Frecuencia absoluta, \% $=$ & Frecuencia relativa, DE= Desviación estándar.
\end{tabular}

La validación de las propiedades métricas del instrumento incluye:

Validez de constructo.

El análisis de variabilidad de los ítems, reveló que la capacidad discriminante de los ítems, es en general muy elevada encontrando índices de variabilidad superiores a 0,53 ; con una mediana de 0,63 ; se constata que todos los ítems son muy homogéneos en términos de varianza, siendo adecuados para discriminar entre sujetos con valores distintos en la variable medida (Tabla 2 ).

El análisis de la matriz de correlación (Coeficiente de Pearson), reveló correlaciones positivas entre los ítems, con valores de correlación ítems-total, desde 0,45 a 0,70 $(p<0,00)$. (Tabla 2).

Tabla 2. Análisis de la variabilidad de los ítems y matriz de correlación.

\begin{tabular}{|c|c|c|c|}
\hline $\mathrm{N}^{\circ}$ & Ítems & Varianza & $\begin{array}{l}\text { Correlación } \\
\text { (Suma) }\end{array}$ \\
\hline 1 & Creo que en la vida todo tiene un sentido profundo. & 0,57 & 0,63 \\
\hline 2 & $\begin{array}{l}\text { No importa el lugar o circunstancia, siempre actúo de acuerdo a } \\
\text { mis principios. }\end{array}$ & 0,56 & 0,73 \\
\hline 3 & $\begin{array}{l}\text { Mis momentos de práctica espiritual me renuevan las fuerzas } \\
\text { físicas. }\end{array}$ & 0,66 & 0,64 \\
\hline 4 & $\begin{array}{l}\text { Cuando pienso en el milagro de mi existencia me lleno de } \\
\text { alegría. }\end{array}$ & 0,59 & 0,61 \\
\hline 5 & $\begin{array}{l}\text { Cuando estoy abocado a la misión noble de mi práctica laboral } \\
\text { (profesión) mis fuerzas se multiplican. }\end{array}$ & 0,63 & 0,71 \\
\hline 6 & En mi tiempo libre disfruto de la naturaleza & 0,65 & 0,64 \\
\hline 7 & $\begin{array}{l}\text { Mi mente se apacigua cuando reflexiono sobre algún texto } \\
\text { espiritual }\end{array}$ & 0,53 & 0,59 \\
\hline 8 & $\begin{array}{l}\text { Creo que cuidar mi cuerpo y de los pacientes es un deber } \\
\text { sagrado. }\end{array}$ & 0,56 & 0,68 \\
\hline 9 & $\begin{array}{l}\text { Cuando experimento un fracaso, todavía puedo encontrar } \\
\text { significado en él. }\end{array}$ & 0,62 & 0,62 \\
\hline 10 & $\begin{array}{l}\text { Frecuentemente reflexiono sobre el significado de los } \\
\text { acontecimientos en mi vida. }\end{array}$ & 0,71 & 0,59 \\
\hline 11 & $\begin{array}{l}\text { Cuando un paciente me necesita siempre me hago un tiempo } \\
\text { para ayudar. }\end{array}$ & 0,60 & 0,67 \\
\hline 12 & $\begin{array}{l}\text { Me defino a mí mismo por mi ser más profundo y no por mi ser } \\
\text { físico. }\end{array}$ & 0,58 & 0,57 \\
\hline 13 & $\begin{array}{l}\text { Soy capaz de reflexionar profundamente acerca de lo que puede } \\
\text { haber más allá de la muerte. }\end{array}$ & 0,72 & 0,51 \\
\hline 14 & $\begin{array}{l}\text { Más allá del plano humano, hay un Ser superior con el cual nos } \\
\text { podemos relacionar. }\end{array}$ & 0,65 & 0,67 \\
\hline 15 & Frecuentemente veo las situaciones y opciones de forma más & 0,75 & 0,54 \\
\hline
\end{tabular}


clara cuando medito, oro o rezo.

Soy consciente de que existe una conexión más profunda entre otras personas y yo.

$0,77 \quad 0,53$

Estoy seguro que ayudar a los demás o ser solidario con los pacientes es mi misión en esta vida.

$0,64 \quad 0,67$

Es difícil para mí pensar en algo más haya que el mundo físico y material.

$0,80 \quad 0,45$

SUMA

77,27

Se realizó el Análisis Factorial Exploratorio y el test de esfericidad de Bartlett arrojo un estadístico de 1170,2; con un $p=0,000$; la medida de adecuación muestral KMO fue de 0,902; lo cual aconseja su factorización ya que nos permiten afirmar que existen correlaciones significativas entre las variables; el Análisis Factorial Confirmatorio, reveló una estructura de tres factores, que explicaron el $54,04 \%$ de la varianza total del cual $38,27 \%$ lo explica el factor $1 ; 8,57 \%$ el factor 2 y $7,20 \%$ el factor 3 (Tabla 3); de este modo se observa además en el gráfico de sedimentación, que el factor 1 , aporta la mayor variabilidad, mostrando la proporción de varianza que explica cada uno de los componentes en el resultado total (Figura 1).

Tabla 3. Análisis factorial confirmatorio - AFC

\begin{tabular}{ccccccc}
\hline & \multicolumn{3}{c}{ Autovalores iniciales } & \multicolumn{2}{c}{$\begin{array}{c}\text { Sumas de las saturaciones al } \\
\text { cuadrado de la extracción }\end{array}$} \\
\cline { 2 - 6 } Componente & Total & $\begin{array}{c}\text { \% de la } \\
\text { varianza }\end{array}$ & $\begin{array}{c}\% \\
\text { acumulado }\end{array}$ & Total & $\begin{array}{c}\text { \% de la } \\
\text { varianza }\end{array}$ & $\begin{array}{c}\% \\
\text { acumulado }\end{array}$ \\
\hline 1 & 6,89 & 38,27 & 38,27 & 6,89 & 38,27 & 38,27 \\
2 & 1,54 & 8,57 & 46,84 & 1,54 & 8,57 & 46,84 \\
3 & 1,30 & 7,20 & 54,04 & 1,30 & 7,20 & 54,04 \\
4 & 0,95 & 5,25 & 59,29 & & \\
5 & 0,87 & 4,81 & 64,09 & & \\
6 & 0,74 & 4,12 & 68,22 & & \\
7 & 0,69 & 3,84 & 72,05 & & \\
8 & 0,65 & 3,61 & 75,66 & & \\
9 & 0,61 & 3,38 & 79,04 & & \\
10 & 0,56 & 3,11 & 82,15 & & \\
11 & 0,52 & 2,88 & 85,03 & & \\
12 & 0,50 & 2,80 & 87,83 & & \\
13 & 0,47 & 2,59 & 90,41 & & \\
14 & 0,44 & 2,42 & 92,83 & & \\
15 & 0,39 & 2,15 & 94,98 & & & \\
16 & 0,34 & 1,86 & 96,84 & & & \\
17 & 0,31 & 1,73 & 98,57 & & \\
18 & 0,26 & 1,43 & 100 & & \\
\end{tabular}


Figura 1. Gráfico de sedimentación posterior al análisis de componentes principales con los 18 items incluidos.

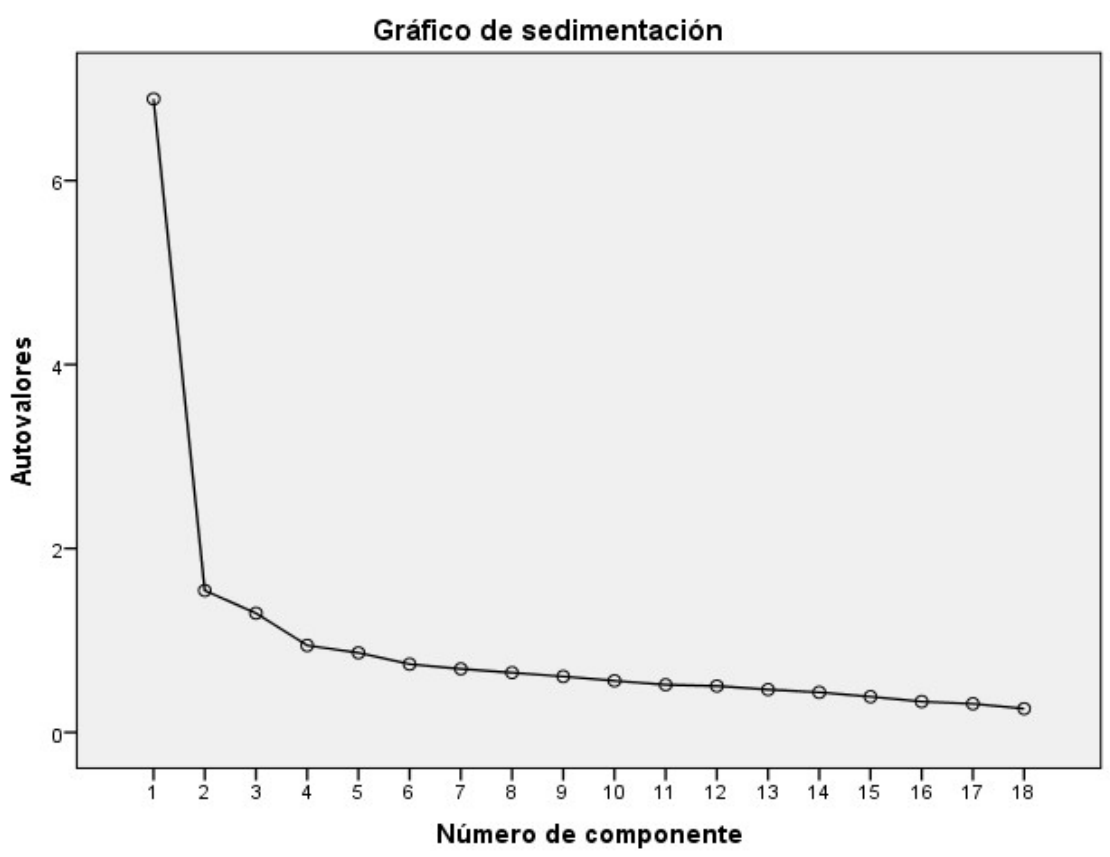

En la tabla 4 se presentan las cargas factoriales obtenidas por los 18 reactivos de la versión final de la EIEps, cuya distribución por dimensiones fue la siguiente: 6 reactivos en el primer factor, 7 en el segundo y 5 en el tercero. Al analizar la agrupación de los reactivos por su carga factorial y contrastarlos con el modelo teórico propuesto por el autor, se determinó que los reactivos agrupados en el primer factor $(2,5,8,11,14$ y 17) pertenecen a la dimensión Vivencia Espiritual en la Practica (VEP); el segundo factor agrupo a los reactivos $1,4,7,10,13,16$ y 18 que pertenecen a la dimensión Pensamiento Existencial (PE); en el tercer factor se encuentran los reactivos $3,6,9,12$ y 15 que corresponden a la dimensión Consciencia Trascendental (CT), sub escalas que serán conceptualizados más adelante.

Tabla 4. Cargas factoriales de reactivos en los tres factores de la EIEps

\begin{tabular}{|c|c|c|c|c|}
\hline $\mathrm{N}^{\circ}$ & Items & Factor 1 & $\begin{array}{l}\text { Factor } \\
2\end{array}$ & Factor 3 \\
\hline 2 & $\begin{array}{l}\text { No importa el lugar o circunstancia, siempre actúo } \\
\text { de acuerdo a mis principios. }\end{array}$ & ,748 & & \\
\hline 5 & $\begin{array}{l}\text { Cuando estoy abocado a la misión noble de mi } \\
\text { práctica laboral (profesión) mis fuerzas se } \\
\text { multiplican. }\end{array}$ & ,731 & & \\
\hline 8 & $\begin{array}{l}\text { Creo que cuidar mi cuerpo y de los pacientes es } \\
\text { un deber sagrado. }\end{array}$ & 690 & & \\
\hline 11 & $\begin{array}{l}\text { Cuando un paciente me necesita siempre me } \\
\text { hago un tiempo para ayudar. }\end{array}$ & 689 & & \\
\hline 14 & $\begin{array}{l}\text { Más allá del plano humano, hay un Ser superior } \\
\text { con el cual nos podemos relacionar. }\end{array}$ & ,688 & & \\
\hline 17 & $\begin{array}{l}\text { Estoy seguro que ayudar a los demás o ser } \\
\text { solidario con los pacientes es mi misión en esta } \\
\text { vida. }\end{array}$ & ,668 & & \\
\hline 1 & Creo que en la vida todo tiene un sentido & & ,319 & \\
\hline
\end{tabular}


profundo.

4 Cuando pienso en el milagro de mi existencia me lleno de alegría.

Mi mente se apacigua cuando reflexiono sobre

algún texto espiritual los acontecimientos en mi vida.

Soy capaz de reflexionar profundamente acerca

de lo que puede haber más allá de la muerte.

18 Es difícil para mí pensar sobre algo más haya que el mundo físico y material.

3 Mis momentos de práctica espiritual me renuevan

6 En mi tiempo libre disfruto de la naturaleza

9 Cuando experimento un fracaso, todavía puedo encontrar significado en él.

Me defino a mí mismo por mi ser más profundo y

15 Frecuentemente veo las situaciones y opciones de forma más clara cuando medito, oro o rezo.

\section{Fiabilidad.}

El análisis de fiabilidad, se realizó con el Alfa de Cronbach, el cual se calculó mediante el método de la varianza de los ítems; en la tabla 5, se observa los 18 ítems incluidos y el puntaje específico para cada ítem si este fuera retirado, que en todos los casos es mayor a 0,89. Asimismo, la correlación elemento-total corregida fue superior a 0,4 con excepción del ítem 18. La consistencia interna global fue alta (Alfa $=0,90)$, así como para la dimensión VEP $(0,84)$, PE $(0,76)$ y la dimensión CT $(0,72)$; lo cual, indica que se trata de un instrumento fiable que hará mediciones estables y consistentes.

Tabla 5. Análisis de consistencia interna mediante coeficiente alfa de Cronbach de la EIEps

\begin{tabular}{ccc}
\hline $\mathrm{N}^{\text {o }}$ & $\begin{array}{c}\text { Correlación } \\
\text { elemento-total } \\
\text { corregida }\end{array}$ & $\begin{array}{c}\text { Alfa si el } \\
\text { ítems se } \\
\text { elimina }\end{array}$ \\
\hline Item01 & 0,57 & 0,89 \\
Item02 & 0,68 & 0,89 \\
Item03 & 0,58 & 0,89 \\
Item04 & 0,55 & 0,90 \\
Item05 & 0,66 & 0,89 \\
Item06 & 0,58 & 0,89 \\
Item07 & 0,53 & 0,90 \\
Item08 & 0,63 & 0,89 \\
Item09 & 0,56 & 0,90 \\
Item10 & 0,52 & 0,90 \\
Item11 & 0,61 & 0,89
\end{tabular}




\begin{tabular}{ccc} 
Item12 & 0,51 & 0,90 \\
Item13 & 0,43 & 0,90 \\
Item14 & 0,62 & 0,89 \\
Item15 & 0,46 & 0,90 \\
Item16 & 0,45 & 0,90 \\
Item17 & 0,62 & 0,89 \\
Item18 & 0,37 & 0,90 \\
\hline
\end{tabular}

Validez concurrente.

Frente a la ausencia de un instrumento que valore la IE en el contexto de la práctica sanitaria, se optó por realizar correlaciones entre el perfil global de Inteligencia Espiritual en la Practica Sanitaria, con sus respectivas dimensiones, Vivencia Espiritual en la Práctica, Pensamiento Existencial y Consciencia Trascendental, encontrando correlaciones significativas y directas entre ellas y la variable principal. (Tabla 6).

Tabla 6. Validez concurrente de la ElEps

\begin{tabular}{llcccc}
\hline & & VEP & PE & CT & EIEps \\
\hline \multirow{2}{*}{ VEP } & R de & & & & \\
& Pearson & & 0,699 & 0,781 & 0,92 \\
& p-valor* & & 0,000 & 0,000 & 0,000 \\
PE de & & & & \\
& Pearson & 0,699 & & 0,71 & 0,882 \\
& p-valor & 0,000 & & 0,000 & 0,000 \\
& R de & & & & \\
CT & Pearson & 0,781 & 0,71 & & 0,914 \\
& p-valor & 0,000 & 0,000 & & 0,000 \\
\hline
\end{tabular}

Elaboración propia, VEP=Vivencia espiritual en la práctica, $\mathrm{PE}=$ Pensamiento existencial, $\mathrm{CT}=$ Consciencia trascendental, EIEps= Escala de inteligencia espiritual en la práctica sanitaria, *Significancia estadística obtenida mediante la prueba de R de Pearson.

\section{DISCUSIÓN}

La revisión de la literatura, confirma que existen escasos instrumentos para medir el constructo IE; la mayoría de ellos fueron desarrollados, validados y/o adaptados en contextos canadienses y/o anglosajones ${ }^{(7,29)}$ y muy pocos latinoamericanos ${ }^{(6)}$, lo que conlleva a una serie de problemas de diversidad cultural en un constructo tan complejo ${ }^{(30)}$.

Por tal razón, urge la necesidad de proponer a la comunidad científica instrumentos fiables y contextualizados que evalúen la IE, en una visión más operativa ${ }^{(9,22)}$.

En ese orden de ideas, el estudio tuvo como objetivo generar una escala de medida respecto a la IE en una muestra de trabajadores de salud, que posea validez de contenido y propiedades métricas aceptables. Esta versión del instrumento facilitará futuras investigaciones sobre el constructo IE en el contexto operativo de la práctica sanitaria y sus posibles asociaciones con otras variables de interés científico. 
Como muestran los resultados de la evaluación de las propiedades métricas; la EIEps presenta una consistencia interna aceptable $(0,90)$, con correlaciones moderadas y significativas entre sus ítems. Las pruebas psicométricas utilizadas para justificar el análisis factorial fueron el test de esfericidad de Bartlett (que evalúa si la matriz de correlaciones es una matriz de identidad) y la medida de adecuación muestral KMO (que compara los coeficientes de correlación observados con los coeficientes de correlación parcial). En nuestro estudio, el test de esfericidad de Barlett, tuvo un valor significativo y la medida de adecuación muestral $\operatorname{KMO}(0,902)$. En base a estos dos resultados favorables, se procedió al análisis factorial de la EIEps, el cual confirmó el supuesto teórico según el cual se basó el diseño de la escala (03 dimensiones), donde la dimensión 1 explica la mayor parte de la varianza recogida respecto a la dimensión 2 y dimensión 3. Resumiendo, nos encontramos con una estructura factorial consistente con ítems que pueden medir adecuadamente la variable estudiada; que además cuenta con suficiente evidencia científica ${ }^{(4-10)}$ que apoye la IE en la práctica sanitaria, como constructo claramente definido.

El instrumento estructurado, consta de 18 items, distribuidos en tres dimensiones: Vivencia espiritual en la práctica (VEP) referida a la coherencia conductual de la IE, es decir a los comportamientos virtuosos en el quehacer de la práctica sanitaria (Items 2 , 5, 8, 11, 14 y 17 ); Pensamiento existencial (PE), implica la capacidad de reflexionar sobre la existencia del ser humano, significado, propósito en la vida y otras cuestiones existenciales, como la realidad, el universo, la muerte, entre otros, (Ítems 1, 4, 7, 10, 13, 16 y 18) y Consciencia trascendental (CT), referida a la capacidad de tomar conciencia, que existe una divinidad o ser superior y que somos más que el cuerpo físico, mental y emocional. (Items 3, 6, 9, 12 y 15); con cuatro opciones de respuesta (Nada verdadero para mí, Algo verdadero para mí, Bastante verdadero para mí, Totalmente verdadero para mí), con puntaje del 1 al 4.

La EIEps, tiene una puntuación global en forma numérica, con calificaciones que van desde los 18 hasta los 72 puntos, donde el puntaje <45 equivale a una IE No saludable, de 45-58 IE por mejorar y >58 puntos IE saludable; del mismo modo para obtener los puntos de corte para las sub escalas se recomienda emplear la técnica de Estanino. No es el objetivo de la escala global ni de las dimensiones tener una función discriminante. El instrumento de medición documental mide una variable subjetiva por lo tanto puede variar de acuerdo al contexto donde se aplique.

Estamos convencidos que la $\mathrm{IE}$, en el contexto de la práctica sanitaria, es la única alternativa para humanizar los servicios de salud; promover el desarrollo y cultivar este tipo de inteligencia, permite mejorar la calidad de la atención y gestión; de ahí, que generar un instrumento de medición con esa finalidad resulta relevante en estos tiempos de crisis de valores, carencia moral y falta de ética, como pautas y paradigmas de la sociedad que tenemos hoy en día. Humanizar para tener establecimientos sanitarios de calidad y un sistema de salud más efectivo es política sectorial; en consecuencia, una estrategia que contribuya a ese propósito debe ser implementada en forma real y sostenida.

En cuanto a las limitaciones del estudio, consideramos que el instrumento de medición documental generado mide una variable subjetiva, la cual se trata de un pensamiento abstracto o superior, sin embargo, es importante como medida de IE, toda vez que, desarrollar y cultivar IE, es más necesaria que nunca, en estos tiempos de crisis reflejada en la deshumanización de la sociedad. Estudiar esta variable particularmente en el ámbito sanitario, nos permitiría conocer una realidad pocas 
veces explorada y en función a los resultados planificar y ejecutar acciones y/o planes de mejora, con la finalidad de contribuir a humanizar los servicios de salud. Por otro lado, la falta de sinceridad por parte de algunos sujetos a la hora de emitir respuestas, constituye una limitación importante, sin embargo, esta puede ser superada con un adecuado control de calidad de las fichas y una correcta aplicación de la encuesta heteroadministrada; finalmente la utilización de una muestra recopilada de forma accidental y no aleatoria, puede ser considerada como una debilidad.

\section{CONCLUSIÓN}

Los resultados obtenidos de la EIEps demuestran índices de confiabilidad y validez dentro de lo esperado; esto fue evidenciado al observar la consistencia interna del instrumento final. De igual forma, la validez de constructo del instrumento fue examinada a través de un análisis de variabilidad, correlación y factores confirmatorio, las propiedades psicométricas de la escala final demuestran que el instrumento es apropiado, sin embargo, puede ser mejorado, por lo tanto se recomienda su uso en futuras investigaciones.

Recomendamos administrar la EIEps, a otra muestra de participantes para realizar el proceso de validación cruzada que incluya un análisis de factores confirmatorio con el propósito de examinar la estructura factorial identificada en este estudio, además de un análisis concurrente (validez de criterio) con otros instrumentos de medición documental; finalmente recomendamos que la EIEps, se valide en poblaciones de otros países, para auscultar sus propiedades psicométricas.

\section{REFERENCIAS}

1. Gardner H. Frames of Mind. The Theory of Multiple Intelligences. Nueva York, Basic Books. (Versión castellana (2001): Estructuras de la Mente. La Teoría de las Inteligencias Múltiples. México, FCE; 1983.

2. Gardner H. Intelligence Reframed: Multiple Intelligences for the 21st Century. Nueva York, Basic Books. (Versión castellana (2001): La Inteligencia Reformulada. Las Inteligencias Múltiples en el Siglo XXI. Barcelona, Paidos; 1999.

3. Torralba F. La Inteligencia Espiritual. Edit. Plataforma Actual. $3^{\circ}$ Edición. Barcelona, España; 2010.

4. Amram Y. The seven dimensions of spiritual intelligence: An ecumenical, grounded theory. Paper presented at the 115th Annual Conference of the American Psychological Association, San Francisco, California, USA. [Internet]. Retrieved from; 2007. [citado el 9 de diciembre de 2019]. Disponible en: http://www.yosiamram.net/docs/7_Dimensions_of_SI_APA_confr_paper_Yosi_Amram .pdf

5. Emmons R. Is spirituality an intelligence. The International Journal for the Psychology of Religion. 2000; 10(1): 3-26. DOI:10.1207/S15327582IJPR1001_2

6. Golovina $O$. The spiritual intelligence as an integrating factor of human consciousness. Zenodo. 2016; 52, 44-52. DOI:10.5281/zenodo.44709

7. King DB, DeCicco TL. A viable model and self-report measure of spiritual intelligence. The International Journal of Transpersonal Studies. 2009; 28, 68-85. DOI:10.24972/ijts.2009.28.1.68

8. Mahasneh AM, Shammout NA, Alkhazaleh ZM, Al-Alwan AF, Abu-Eita JD. The relationship between spiritual intelligence and personality traits among Jordanian 
university students. Psychol Res Behav Manag. 2015; 8: 89-97. DOI: 10.2147/PRBM.S76352

9. Wolman R. Thinking with your soul. Spiritual intelligence and why it matters. New York, NY: Harmony Books; 2001.

10. Zohar D, Marshall I. Spiritual intelligence, the ultimate intelligence, SQ. London, UK: Bloomsbury; 2000.

11. King D. B. (2008). Rethinking claims of spiritual intelligence: A definition, model, \& measure. Unpublished master's thesis, Trent University, Peterborough, ON, Canada 2008. [citado el 12 de diciembre de 2019]. Disponible en: https://docplayer.net/4796647-Rethinking-claims-of-spiritual-intelligence-a-definitionmodel-and-measure.html

12. Emmons R. The psychology of ultimate concerns. New York: Guilford Press; 1999.

13. Halama P, Striženec M. Spiritual, existential or both? Theoretical considerations on the nature of "higher" intelligences. Studia Psychologica [Internet].2004 [citado el 15 de diciembre de 2019]; 46(3): 239-253. Disponible en: https://www.researchgate.net/publication/288446346_Spiritual_existential_or_both_Th eoretical_considerations_on_the_nature_of_higher_intelligences

14. Antunes $R$, Silva $\bar{A}$, Oliveira J. Spiritual Intelligence Self-Assessment Inventory: Psychometric properties of the Portuguese version of SISRI-24, Journal of Religion, Spirituality \& Aging. 2017; 30: 12-14 DOI: 10.1080/15528030.2017.1324350

15. Kwilecki S. Spiritual intelligence as a theory of individual religion: A case application, The International Journal for the Psychology of Religion. 2000; 10(1): 3546. https://doi.org/10.1207/S15327582IJPR1001_4

16. Emmons R. The psychology of ultimate concerns. New York: Guilford Press; 1999.

17. Bonilla E. Conexión mente-cuerpo, fenómenos parapsicológicos y curación espiritual. Invest Clin. 2010; 51(2): 209-238. Recuperado de: http://ve.scielo.org/scielo.php?script=sci arttext\&pid=S0535-51332010000200005

18. Carrillo E. Amor: Vida y Consciencia. 2012. Recuperado de: https://despertadhumanidad.wordpress.com/2012/12/10/amor-vida-y-conscienciaemilio-carrillo-libro-gratis/

19. Hall D, Meador $\mathrm{K}$, Koenig $\mathrm{H}$. Measuring religiousness in health research: review and critique. Journal of Religion and Health. 2008; 47(2): 134-163. DOI: 10.1007/s10943-008-9165-2.

20. Fetzer Institute/National Institute on Aging Working Group. (1999). Multidimensional Measurement of Religiousness/Spirituality for Use in Health Research [Internet].Kalamazoo, Michigan: Fetzer Institute; 1999 [citado el 18 de diciembre de 2019]. Disponible en: https://www.gem-measures.org , DownloadMeasure

21. Baldacchino DR, Buhagiar A. Psychometric evaluation of the Spiritual Coping Strategies scale in English, Maltese, backtranslation and bilingual versions. Journal of Advanced Nursing. 2003; 42(6):558-570. DOI: 10.1046/j.1365-2648.2003.02659.x

22. Amram $Y$. The contribution of emotional and spiritual intelligences to effective business leadership (Unpublished doctoral dissertation). Institute of Transpersonal Psychology, Palo Alto, CA. 2009. [citado el 22 de diciembre de 2019]. Disponible en: https://www.intelligensi.com/assets/documents/EI_and_SI_in_Leadership_Amram_Dis sert.pdf

23. Mayta-Tristán P, Mezones-Holguín E, Pereyra-Elías R, Montenegro-Idrogo JJ, Mejia CR, Dulanto-Pizzorni A, et al. Diseño y validación de una escala para medir la percepción sobre el trabajo en el primer nivel de atención en estudiantes de medicina 
de Latinoamérica. Rev Peru Med Exp Salud Pública. 2013;30(2):190-6. DOI: http://dx.doi.org/10.17843/rpmesp.2013.302.190

24. Supo J. Validación de instrumentos de medición documentales, curso en vídeo para descarga inmediata [Internet]. Arequipa-Perú: Bioestadistico EIRL @ 2010 - 2016 [citado el 18 de diciembre de 2019]; [aprox. 1 pantalla]. Disponible en: https://validaciondeinstrumentos.com/carta

25. Supo J. Cómo validar un instrumento - La guía para validar un instrumento en 10 pasos [Internet]. Perú: Bioestadístico; 2013. [citado el 12 de diciembre de 2019]. Disponible en: http://www.cua.uam.mx/pdfs/coplavi/s_p/doc_ng/validacion-deinstrumentos-de-medicion.pdf

26. Barbero M.I. Psicometría II. Métodos de elaboración de escalas. Madrid: Uned. 1993.

27. Pett M, Lackey N, Sullivan J. Making sense of factor analysis: the use of factor analysis for instrument development in health care research. California: Sage Publication; 2003. DOI: https://dx.doi.org/10.4135/9781412984898

28. Campo-Arias A, Oviedo HC. Propiedades psicométricas de una escala: La consistencia interna. Revista de Salud Pública [Internet]. 2008 [citado el 12 de diciembre de 2019]; 10(5), 831-839. Disponible en: https://www.redalyc.org/pdf/422/42210515.pdf

29. Skrzypińska K. Polish adaptation of Spiritual Intelligence Self-Report Inventory (SISRI-24) [Internet]. Polonia: ResearchGate; 2016. [citado el 12 de diciembre de 2019].

Disponible

en: https://www.researchgate.net/publication/308097574_Polish_adaptation_of_Spiritual_I ntelligence_Self-Report_Inventory_SISRI-24

30. Arias $\bar{R}$, Lemos $\bar{V}$. Una aproximación teórica y empírica al constructo de inteligencia espiritual. Enfoques [Internet]. 2017 [citado el 22 de diciembre de 2019]; 27(1), 79-102. Disponible en: http://www.scielo.org.ar/scielo.php?script=sci_arttext\&pid=S1669-

$27212015000100005 \&$ Ing=es\&tlng=es 


\section{ANEXO}

\section{ESCALA DE INTELIGENCIA ESPIRITUAL EN LA PRÁCTICA SANITARIA}

Para cada elemento, marque con una $\mathbf{x}$, la respuesta que mejor le describa. Las cinco respuestas posible son:

\begin{tabular}{|c|c|c|c|}
\hline NV & AV & BV & TV \\
\hline $\begin{array}{c}\text { Nada verdadero } \\
\text { para mí }\end{array}$ & $\begin{array}{c}\text { Algo verdadero } \\
\text { para mí }\end{array}$ & $\begin{array}{c}\text { Bastante } \\
\text { verdadero para } \\
\text { mí }\end{array}$ & $\begin{array}{c}\text { Totalmente verdadero } \\
\text { para mí }\end{array}$ \\
\hline
\end{tabular}

\begin{tabular}{|c|c|c|c|c|c|}
\hline $\mathrm{N}^{0}$ & Items & NV & AV & BV & TV \\
\hline 1 & Creo que en la vida todo tiene un sentido profundo. & & & & \\
\hline 2 & $\begin{array}{l}\text { No importa el lugar o circunstancia, siempre actúo de acuerdo a mis } \\
\text { principios. }\end{array}$ & & & & \\
\hline 3 & Mis momentos de práctica espiritual me renuevan las fuerzas físicas. & & & & \\
\hline 4 & Cuando pienso en el milagro de mi existencia me lleno de alegría. & & & & \\
\hline 5 & $\begin{array}{l}\text { Cuando estoy abocado a la misión noble de mi práctica laboral (profesión) } \\
\text { mis fuerzas se multiplican. }\end{array}$ & & & & \\
\hline 6 & En mi tiempo libre disfruto de la naturaleza & & & & \\
\hline 7 & Mi mente se apacigua cuando reflexiono sobre algún texto espiritual & & & & \\
\hline 8 & Creo que cuidar mi cuerpo y de los pacientes es un deber sagrado. & & & & \\
\hline 9 & Cuando experimento un fracaso, todavía puedo encontrar significado en él. & & & & \\
\hline 10 & $\begin{array}{l}\text { Frecuentemente reflexiono sobre el significado de los acontecimientos en mi } \\
\text { vida. }\end{array}$ & & & & \\
\hline 11 & Cuando un paciente me necesita siempre me hago un tiempo para ayudar. & & & & \\
\hline 12 & Me defino a mí mismo por mi ser más profundo y no por mi ser físico. & & & & \\
\hline 13 & $\begin{array}{l}\text { Soy capaz de reflexionar profundamente acerca de lo que puede haber más } \\
\text { allá de la muerte. }\end{array}$ & & & & \\
\hline 14 & $\begin{array}{l}\text { Más allá del plano humano, hay un Ser superior con el cual nos podemos } \\
\text { relacionar. }\end{array}$ & & & & \\
\hline 15 & $\begin{array}{l}\text { Frecuentemente veo las situaciones y opciones de forma más clara cuando } \\
\text { medito, oro o rezo. }\end{array}$ & & & & \\
\hline 16 & $\begin{array}{l}\text { Soy consciente de que existe una conexión más profunda entre otras } \\
\text { personas y yo. }\end{array}$ & & & & \\
\hline 17 & $\begin{array}{l}\text { Estoy seguro que ayudar a los demás o ser solidario con los pacientes es mi } \\
\text { misión en esta vida. }\end{array}$ & & & & \\
\hline 18 & Es difícil para mí pensar en algo más allá que el mundo físico y material. & & & & \\
\hline
\end{tabular}

Gracias por su colaboración

ISSN 1695-6141

(c) COPYRIGHT Servicio de Publicaciones - Universidad de Murcia 